\title{
Recurrent Hyperparathyroidism Following Successful Four- Gland Parathyroidectomy and Normalization of Parathyroid Hormone in a Renal Transplant Patient
}

\author{
Ryan Petruccia, d, David Johnston $^{\mathrm{b}}$, Tamara Preda ${ }^{\mathrm{a}, \mathrm{c}}$
}

\begin{abstract}
Chronic kidney disease has an estimated prevalence of $10 \%$ in Australia and is predicted to rise in the coming years. Secondary hyperparathyroidism resulting from chronic kidney disease is an important cause of morbidity in these patients; and screening for secondary hyperparathyroidism is recommended in international guidelines. We present the case of a chronic kidney disease patient who developed recurrent hyperparathyroidism despite previous "total" parathyroidectomy and subsequent renal transplant. After targeted investigations he was diagnosed with an accessory parathyroid gland in his thorax, causing the recurrent hyperparathyroidism. He was managed with a thoracoscopic excision with a resultant drop in parathyroid hormone consistent with surgical cure. This case highlights the rare phenomenon of supernumerary and ectopic parathyroid glands. Cross sectional thoracic imaging can and should be used to detect and localize supernumerary glands not apparent at the time of original surgery.
\end{abstract}

Keywords: Supernumerary parathyroid; Secondary hyperparathyroidism; Parathyroidectomy; Ectopic parathyroid

\section{Introduction}

According to the Australian Bureau of Statistics 2011-12 Australian Health Survey, an estimated $10 \%$ of Australian adults over the age of 18 years had biomedical signs of chronic kidney disease (CKD) [1]. The Kidney Disease: Improving Global Outcomes guidelines recommend that patients with stage 3

Manuscript submitted January 9, 2021, accepted January 20, 2021

Published online February 8, 2021

${ }^{a}$ Liverpool Hospital Department of Endocrine, Head and Neck Surgery, Elizabeth Street, Liverpool, NSW 2170, Australia

${ }^{\mathrm{b}}$ Clearview Medical Imaging, Station Street, Fairfield, NSW 2165, Australia 'School of Medicine, University of Notre Dame, Oxford Street, Darlinghurst, Sydney, NSW 2010, Australia

${ }^{\mathrm{d} C}$ Corresponding Author: Ryan Petrucci, Liverpool Hospital Department of Endocrine, Head and Neck Surgery, Elizabeth Street, Liverpool, NSW 2170, Australia. Email: r_petrucci@hotmail.com

doi: https://doi.org/10.14740/jmc3643
CKD undergo screening for secondary hyperparathyroidism (SHPT) [2].

Endocrine and biochemical anomalies associated with renal hyperparathyroidism relate to bone mineral/metabolic and cardiovascular disease. SHPT in CKD patients is associated with increased fractures, hyperphosphatemia, anemia, vascular and tissue calcification; these are important to treat before renal transplantation [3]. There is also some evidence to suggest hypertension improves post parathyroidectomy in these patients, and there is a further benefit of parathyroidectomy prior to renal transplant [4].

Medical management for SHPT can be divided into pharmacological and surgical options. Pharmacological agents include vitamin $\mathrm{D}$, calcimimetics and phosphorus binders. Surgical options include total and subtotal parathyroidectomy [3]. Internationally used guidelines such as the National Kidney Foundation Kidney Disease Outcome Quality Initiative (KDOQI) recommend parathyroidectomy for patients who are refractory to medical management [5].

\section{Case Report}

A 62-year-old man was referred by his treating physicians to an endocrine surgeon with presumed tertiary hyperparathyroidism. He had a history of total parathyroidectomy and bilateral cervical thymectomy 17 years prior for SHPT with underlying chronic renal failure due to immunoglobulin $\mathrm{A}(\operatorname{Ig} \mathrm{A})$ nephropathy as a child.

Review of the histopathology report from this surgery confirmed removal of four hyperplastic parathyroid glands weighing between $0.285-0.815 \mathrm{~g}$.

The operation report noted four parathyroid glands in their expected locations in the neck. Comment was made that bilateral inferior parathyroids were found in the thyrothymic tract and were noted to be larger than their superior counterparts. The lower limit of dissection was the left brachiocephalic vein posterior to the manubrium (demarcated by surgical clips).

Following his original surgery, parathyroid hormone (PTH) dropped from $93 \mathrm{pmol} / \mathrm{L}$ to $4.6 \mathrm{pmol} / \mathrm{L}$ with laboratory reference range (RR) $1.6-6.9 \mathrm{pmol} / \mathrm{L}$. This result was consistent with biochemical cure.

Five years after parathyroid surgery the patient underwent a renal transplant with excellent renal function for 8 years. His 


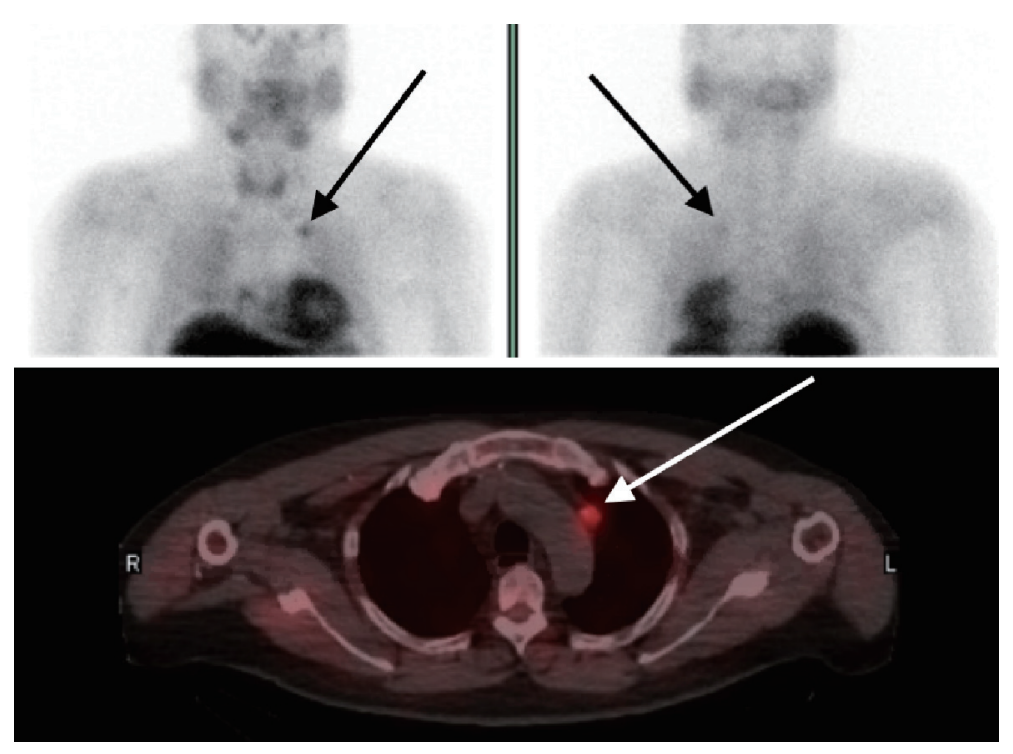

Figure 1. Sestamibi parathyroid scan showing uptake in a mediastinal ectopic parathyroid gland situated adjacent to the arch of the aorta, highlighted by the black and white arrows.

renal function then deteriorated culminating in a return to peritoneal dialysis (oliguric but not anuric). At the time of surgical review laboratory test results showed a PTH $120 \mathrm{pmol} / \mathrm{L}$ (RR 1.6 - $6.9 \mathrm{pmol} / \mathrm{L})$, corrected serum calcium $2.60 \mathrm{mmol} / \mathrm{L}(\mathrm{RR}$ $2.15-2.55 \mathrm{mmol} / \mathrm{L})$ and estimated glomerular filtration rate (eGFR) $7 \mathrm{~mL} / \mathrm{min} / 1.73 \mathrm{~m}^{2}$. These results were indicative of tertiary hyperparathyroidism.

Imaging investigations were undertaken in order to localize the functioning parathyroid tissue responsible for elevation of PTH levels. A neck ultrasound did not detect any mass lesions. A Sestamibi single photon-emission computed tomography (SPECT)/computed tomography $(\mathrm{CT})$ reported no focal uptake in or around the thyroid. A 12-mm thoracic para-aortic nodule was seen in the left anterior/superior mediastinum adjacent to the aortic arch with moderate focal uptake (Fig. 1).

The patient was referred for four-dimensional (4D) para-

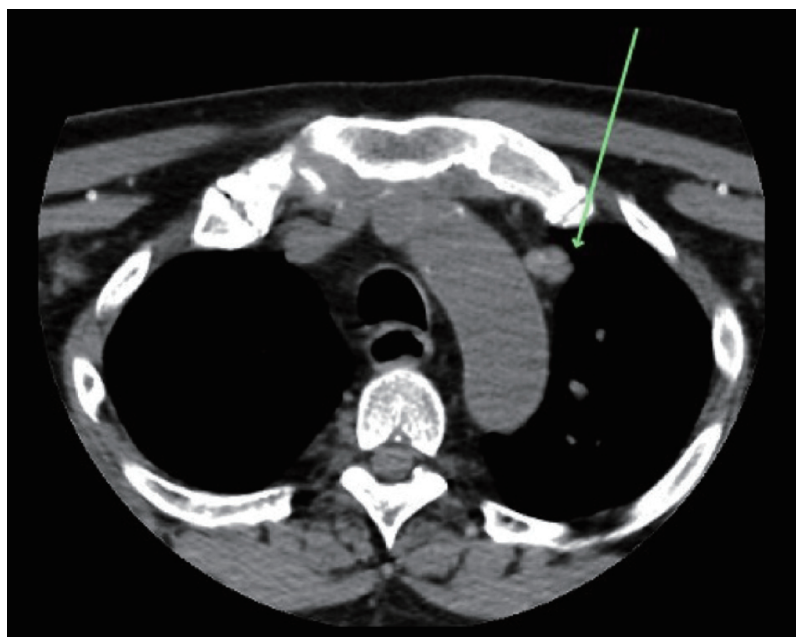

Figure 2. Axial CT image of mediastinal mass highlighted with the green arrow. CT: computed tomography. thyroid CT. Non-contrast images were obtained given the patient was not anuric. A mildly lobulated rounded lesion adjacent to the aortic arch in the superior mediastinum measuring $15 \times 11 \mathrm{~mm}$ with a single punctate focus of calcification was reported (Figs. 2, 3).

The patient was referred on to a cardiothoracic surgeon and a thoracoscopic excision of the lesion was undertaken in

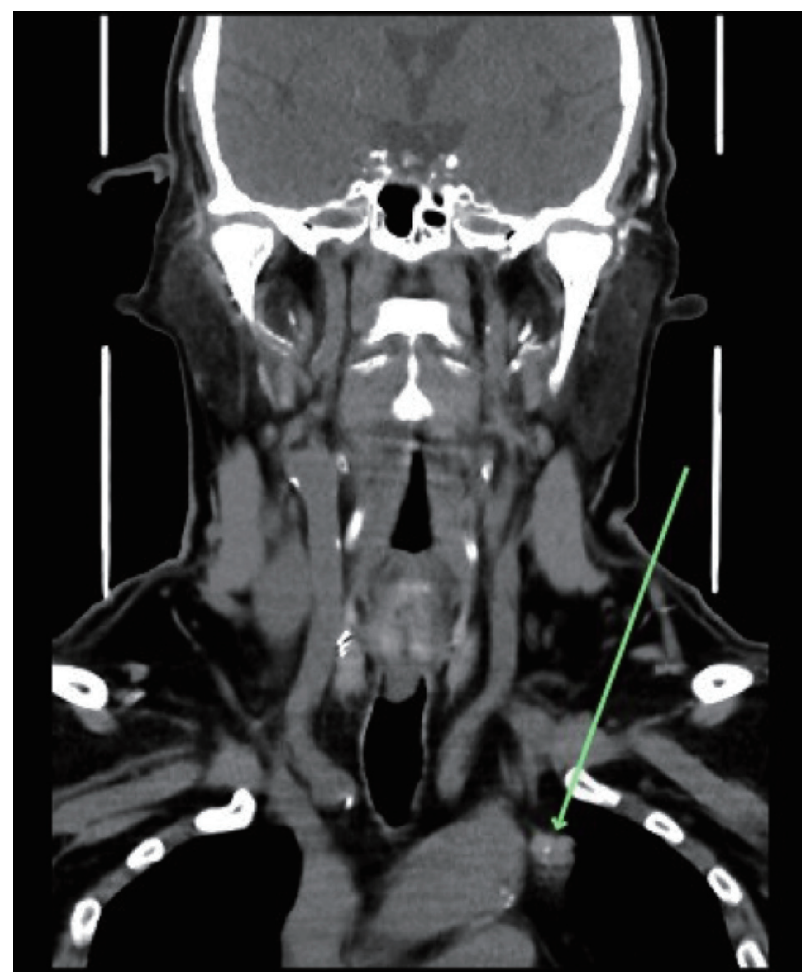

Figure 3. Coronal CT image of mediastinal mass highlighted with the green arrow. CT: computed tomography. 
March 2020. The tumor was located in accordance with the imaging on the left side of the aortic arch and noted to be intimately associated with the phrenic nerve.

The surgical specimen was sent for histological assessment and reported a 1.7-g fat depleted parathyroid gland with focal fibrosis and hemorrhage in keeping with an adenoma; no atypia.

Post-operative PTH dropped to $18.9 \mathrm{pmol} / \mathrm{L}$ compared to $119 \mathrm{pmol} / \mathrm{L}$, which was taken 2 months prior to surgery. Post-operative corrected serum calcium normalized to 2.19 $\mathrm{mmol} / \mathrm{L}$. He was commenced on calcium and 1,25-dihydroxycholecalciferol supplements to maintain normal corrected serum calcium levels and for treatment of bone disease.

He has been listed for a second renal transplant.

\section{Discussions}

Recurrent hyperparathyroidism although not common is of clinical significance due to its deleterious effects. Recurrence in patients who have undergone total parathyroidectomy has been attributed to incomplete cervical parathyroidectomy or supernumerary glands. A large-scale follow-up study of 519 patients who underwent total parathyroidectomy showed a recurrence rate of $2.4 \%$ with $50 \%$ of these cases diagnosed with supernumerary mediastinal parathyroid glands [6]. These patients required re-do surgery after imaging localization.

Approximately $2-13 \%$ of the population have supernumerary (five or six) parathyroid glands [7, 8]. Ectopic parathyroids are defined as functional parathyroid glands located outside the normal anatomical position due to aberrant migration during fetal development [9]. They are found most commonly in the thymus, within the thyroid or in the mediastinum [6].

In CKD patients with SHPT it can take 6 - 12 months for PTH levels to normalize post transplant [10]. Patients who continue to suffer from SHPT or recurrent disease need proper investigation and either pharmacological or surgical treatment. The current quoted prevalence of parathyroidectomy in post transplant patients is $0.6-5.6 \%$ [10].

In regards to this case it appears that the patient had an adequate PTH response to total parathyroidectomy initially. However, over time recurrent hyperparathyroidism manifested and was determined to be due to an ectopic supernumerary parathyroid gland. Whilst rare, this highlights the need for ongoing surveillance of renal hyperparathyroidism patients with consideration to the presence of supernumerary glands. Dual localization with sestamibi and CT is optimal when a non-neck location is likely.

\section{Learning points}

This case demonstrates that it is worthwhile to follow up patients who have been treated for SHPT, particularly once in receipt of renal transplant. Biochemical surveillance is simple and effective in detecting recurrence. Cross sectional thoracic imaging can and should be used to detect and localize supernumerary glands not apparent at the time of original surgery.

\section{Acknowledgments}

None to declare.

\section{Financial Disclosure}

None to declare.

\section{Conflict of Interest}

None to declare.

\section{Informed Consent}

Informed consent was gained prior to completing the case report.

\section{Author Contributions}

RP and TP both contributed to the conception, writing and editing of the case report. DJ contributed to the radiology figures.

\section{Data Availability}

The authors declare that data supporting the findings of this study are available within the article.

\section{References}

1. Australian Institute of Health and Welfare 2020. Chronic kidney disease. Cat. no. CDK 16. Canberra: AIHW. [Cited 2020 Aug 25]. https://www.aihw.gov.au/reports/ chronic-kidney-disease/chronic-kidney-disease.

2. Kidney Disease Improving Global Outcomes. Executive summary of the 2017 KDIGO Chronic Kidney Disease - Mineral and Bone Disorder (CKD-MBD) Guideline Update: what's changed and why it matters. [Cited 2020 Aug 25]. https://kdigo.org/wp-content/uploads/2017/02/ Executive-Summary-_2017-KDIGO-CKD-MBD-GLUpdate Corrected.pdf.

3. Lau WL, Obi Y, Kalantar-Zadeh K. Parathyroidectomy in the management of secondary hyperparathyroidism. Clin J Am Soc Nephrol. 2018;13(6):952-961.

4. Lorenz K, Bartsch DK, Sancho JJ, Guigard S, Triponez F. Surgical management of secondary hyperparathyroidism in chronic kidney disease - a consensus report of the European Society of Endocrine Surgeons. Langenbecks Arch Surg. 2015;400(8):907-927.

5. Delos Santos R, Rossi A, Coyne D, Maw TT. Management of post-transplant hyperparathyroidism and bone disease. Drugs. 2019;79(5):501-513. 
6. Tominaga Y, Numano M, Tanaka Y, Uchida K, Takagi H. Surgical treatment of renal hyperparathyroidism. Semin Surg Oncol. 1997;13(2):87-96.

7. Wang C. The anatomic basis of parathyroid surgery. Ann Surg. 1976;183(3):271-275.

8. Pattou FN, Pellissier LC, Noel C, Wambergue F, Huglo DG, Proye CA. Supernumerary parathyroid glands: frequency and surgical significance in treatment of renal hyperparathyroidism. World J Surg. 2000;24(11):1330-
1334.

9. Noussios G, Anagnostis P, Natsis K. Ectopic parathyroid glands and their anatomical, clinical and surgical implications. Exp Clin Endocrinol Diabetes. 2012;120(10):604610.

10. Evenepoel P, Claes K, Kuypers DR, Debruyne F, Vanrenterghem Y. Parathyroidectomy after successful kidney transplantation: a single centre study. Nephrol Dial Transplant. 2007;22(6):1730-1737. 